\title{
Nonlinear Modeling of FES-Supported Standing-Up in Paraplegia for Selection of Feedback Sensors
}

\author{
Roman Kamnik, Member, IEEE, Jian Qing Shi, Roderick Murray-Smith, and Tadej Bajd, Fellow, IEEE
}

\begin{abstract}
This paper presents analysis of the standing-up manoeuvre in paraplegia considering the body supportive forces as a potential feedback source in functional electrical stimulation (FES)-assisted standing-up. The analysis investigates the significance of arm, feet, and seat reaction signals to the human body center-of-mass (COM) trajectory reconstruction. The standing-up behavior of eight paraplegic subjects was analyzed, measuring the motion kinematics and reaction forces to provide the data for modeling. Two nonlinear empirical modeling methods are implemented-Gaussian process (GP) priors and multilayer perceptron artificial neural networks (ANN)-and their performance in vertical and horizontal COM component reconstruction is compared. As the input, ten sensory configurations that incorporated different number of sensors were evaluated trading off the modeling performance for variables chosen and ease-of-use in everyday application. For the purpose of evaluation, the root-mean-square difference was calculated between the model output and the kinematics-based COM trajectory.

Results show that the force feedback in COM assessment in FES assisted standing-up is comparable alternative to the kinematics measurement systems. It was demonstrated that the GP provided better modeling performance, at higher computational cost. Moreover, on the basis of averaged results, the use of a sensory system incorporating a six-dimensional handle force sensor and an instrumented foot insole is recommended. The configuration is practical for realization and with the GP model achieves an average accuracy of COM estimation $16 \pm 1.8 \mathrm{~mm}$ in horizontal and $39 \pm 3.7$ $\mathrm{mm}$ in vertical direction. Some other configurations analyzed in the study exhibit better modeling accuracy, but are less practical for everyday usage.
\end{abstract}

Index Terms-Artificial neural network (ANN), center of mass (COM), feedback, functional electrical stimulation (FES), Gaussian process (GP), mixture models, paraplegia, position tracking, standing-up.

Manuscript received February 25, 2004; revised October 5, 2004. This work was supported in part by the Republic of Slovenia Ministry of Education, Science, and Sport under Grant Motion analysis and synthesis in man and machine P2-0228, in part by the EPSRC under Grant Modern statistical approaches to off-equilibrium modeling for nonlinear system control GR/M76379/01, in part by the Science Foundation Ireland under Grant 00/PI.1/C067, and in part by the European Commission under Research Training Network Grant Multi-Agent Control HPRN-CT-1999-00107.

R. Kamnik and T. Bajd are with the Faculty of Electrical Engineering, University of Ljubljana, Ljubljana 1000, Slovenia (e-mail: roman.kamnik@fe.uni-lj.si; tadej.bajd@fe.uni-lj.si).

J. Q. Shi was with the Department of Computing Science, University of Glasgow, Glasgow G12 8QQ, U.K. He is now with the School of Mathematics and Statistics, University of Newcastle, Newcastle NE1 7RU, U.K. (e-mail: J.Q.Shi@newcastle.ac.uk).

R. Murray-Smith is with the Department of Computing Science, University of Glasgow, Glasgow G12 8QQ, U.K. and also with the Hamilton Institute, NUI Maynooth, Ireland (e-mail: rod@dcs.gla.ac.uk).

Digital Object Identifier 10.1109/TNSRE.2004.841879

\section{INTRODUCTION}

$\mathbf{R}$ ISING from a sitting to a standing position is a common daily activity in human living. Individuals experiencing rising difficulties have problems living independently, while their prolonged immobilization results in physiological problems. Spinal-cord-injury patients have particular difficulties in standing-up, due to their lower-limb paralysis. To alleviate this, paraplegic patients are trained how to stand up and compensate for the missing action of their lower extremities during the rehabilitation process. The lifting and stabilizing forces are provided by the arm support, requiring strength and motor skill from the upper body. For support, a walker frame, parallel bars, simple stationary standing frame, or even chair arm rests are normally used. However, people practicing a fully arm supported standing-up risk later complications of the upper limb joints [1].

In addition to the arm support, standing-up in paraplegia can be facilitated by functional electrical stimulation (FES). FES is a method of eliciting the action potential in the nerves innervating the paralyzed muscles. This way, the muscle contractions are artificially evoked and motor functions recovered [2]. Bajd et al. proposed a simple approach to the FES supported standing-up of paraplegic subjects [3]. Within this strategy, which is now widely used in home and clinical practice, the stimulation is based on an open-loop surface stimulation of the knee extensors. In the preparation phase, the paraplegic subject brings his body to an initial pose with the upper body leaning forward, arms almost fully flexed at the elbows and supported by the walker frame, while the hip joints resting in the chair are pulled forward toward the edge of chair as much as possible and feet brought backward. For the start of rising, the stimulation is voluntarily triggered by the subject, and the body is lifted upward from the initial to the extended upright position. As the stimulation of the knee extensors is open-loop and on/off triggered with maximum stimulation amplitudes throughout the rising process, the current method for standing up is suboptimal in terms of the applied forces and torques in the upper and lower extremities [4]. On the other side, at the end of the standing-up, when the knees are almost fully extended, the excessive knee joint torques cause high terminal velocities in the knee joints, which can result in ligament injuries [5].

The disadvantages of the traditional approach have led to the development of new approaches to stimulation control, principally based on closed-loop control theory. In the first place, simple control algorithms have been proposed, such as "bangbang" controllers tracking the reference trajectory in the phase plane of variables. As state variables, the knee-joint angle and angle velocity were used in [5], [6], while in [8], the relationship 
between the knee and hip-joint angle velocities was controlled. In some of these studies, the process of the standing-up was divided into phases and the constant stimulation output provided during the particular phase. The tasks of the phase start event detection and the stimulation amplitude alteration were accomplished by the finite state controller [6], [7]. Linear proportional, integral derivative (PID) and nonlinear fuzzy controllers controlling the knee joint angle have also been proposed [9], [10]. Common to these solutions is that the reference values to the controller were determined corresponding to the standing-up of healthy subjects. More advanced proposals, incorporating the paraplegic subject's volition into the stimulation control during rising, have been given in [11], [12]. In both proposals, the stimulation sequences were determined on the basis of subject's activity, i.e., known subject body position and arm reactions. The approaches have been evaluated experimentally and proved efficient in providing the "patient-driven" nature of the stand-up [13], [14]. However, the difficulty in closed-loop stimulation control is that the information fed back to the controller is supposed to be provided by the sensors, normally goniometers and accelerometers, attached to the subject's body. Mounting, dismounting, and wiring of the sensors is a tedious job and is not practical for daily use.

For this reason, we are proposing a method for assessing the subject's body state during rising based on feedback information acquired in a more practical manner. We have chosen the supportive forces acting at the interaction points with the paraplegic's environment as an alternative feedback source. Seat, foot, and arm reactions can be far more easily measured than joint angles: seat and arm support forces can readily be measured using multidimensional load cells mounted in the arm support frame and the seat. An even more practical alternative for instrumenting the subject's environment, is commercially-available shoe insole sensors for the ground reaction forces. Furthermore, the employment of the natural sensory nerve signals from the feet is expected to be functional in the future [15]. As an objective characterizing the body state during rising we have chosen the total body center-of-mass (COM) motion trajectory. In [16], the position of COM in the sagittal plane was found to be the main controlled variable in sit-to-stand task of a healthy subject. Several other studies have examined the position of the COM relative to the base of support (BOS) during activities such as lifting and the initiation and termination of gait [17], [18]. The studies suggest the relationship between the COM and the center of pressure (COP) is one of the principles of balance control by the central nervous system during activity. Lately, some authors proposed that the horizontal velocity of the COM should also be considered in describing the movements for the control of balance, because it governs the destiny of the horizontal position of the COM over the BOS [19]. However, in the standing-up of paraplegic patient, the body is supported by arms and feet in four points forming the larger BOS than that in bipedal activities. Besides, in movement initiation the horizontal velocities are lower because of the typical starting pose taken prior to rising with the upper body leaned forward. Assuring the stable pose and fall prevention are, therefore, less demanding and are already controlled by the subject's voluntary activity of the upper body. For this reason, balance control is not considered the objective task for the FES system, and the COM velocity becomes less important for feedback. In this study, the trajectory of the COM in the sagittal plane is used to characterize the whole body position and the phase of the process in which in the first phase body segments accelerate anteriorly, in the transition phase decelerate anteriorly and accelerate vertically, and in the third phase achieve standing pose by deceleration in the vertical direction [20]-[22]. The use of the COM trajectory for feedback control is appropriate for continuous and for finite state FES control approaches.

According to Newton's second law, the external forces acting on the body are proportional to the body COM acceleration. Hence, the COM displacement in human transient activities can be estimated by a second time integral of the sum of reaction forces. This method is, however, prone to cumulative integration errors, i.e., drift [23], [24]. To overcome this problem, two nonlinear modeling techniques are implemented in this paper. An ANN model and a GP mixture model were designed for the purpose of mapping the interaction forces to the COM trajectory. In the paper, the model input variable selection, the structure, and the performance evaluation are presented and compared.

\section{METHODS}

A concept of the sensory-driven FES-supported standing-up is presented in Fig. 1. The amplitude and frequency of the knee extensors' stimulation are varied according to the COM position while the body is rising. To exploit the support force signals, it is vital that the model is capable of mapping the reaction forces to the COM trajectory. The objective of this study was to build a model for predicting the COM vertical and horizontal displacements on a basis of a limited number of input signals provided by the artificial force sensors.

\section{A. Data Set}

To provide the representative data set for modeling, we analyzed the standing up maneuver of eight paraplegic patients. Five men and three women participated in the study. Their ages ranged from 17 to 57 years, weights from 58 to $95 \mathrm{~kg}$ and heights from 159 to $185 \mathrm{~cm}$. The sample group included patients with different levels of spinal-cord injury and different experience of FES usage as summarized in Table I. The kinematic and kinetic variables of the standing-up trials were assessed with a specially built measurement setup. The data acquired were used in the model design and evaluation.

1) Measurement Instrumentation: The measuring setup used in the standing-up analysis incorporated two systems, one for determining the forces acting to the human body and the second for measuring the body motion trajectory. An assumption of human body symmetry during the standing-up task was made. Hence, measurements were restricted to the patient's right side and were calculated for the left side. To assess the reaction forces, two measuring frames were built as copies of a wheelchair seat and a conventional walker. The seating frame was instrumented by the use of a six-axis AMTI force plate (AMTI, Inc., Watertown, MA), while the force and torque vectors on the right walker handle were assessed by the six-axis JR3 sensor (JR3, Inc., Woodland, CA) usually utilized as wrist 


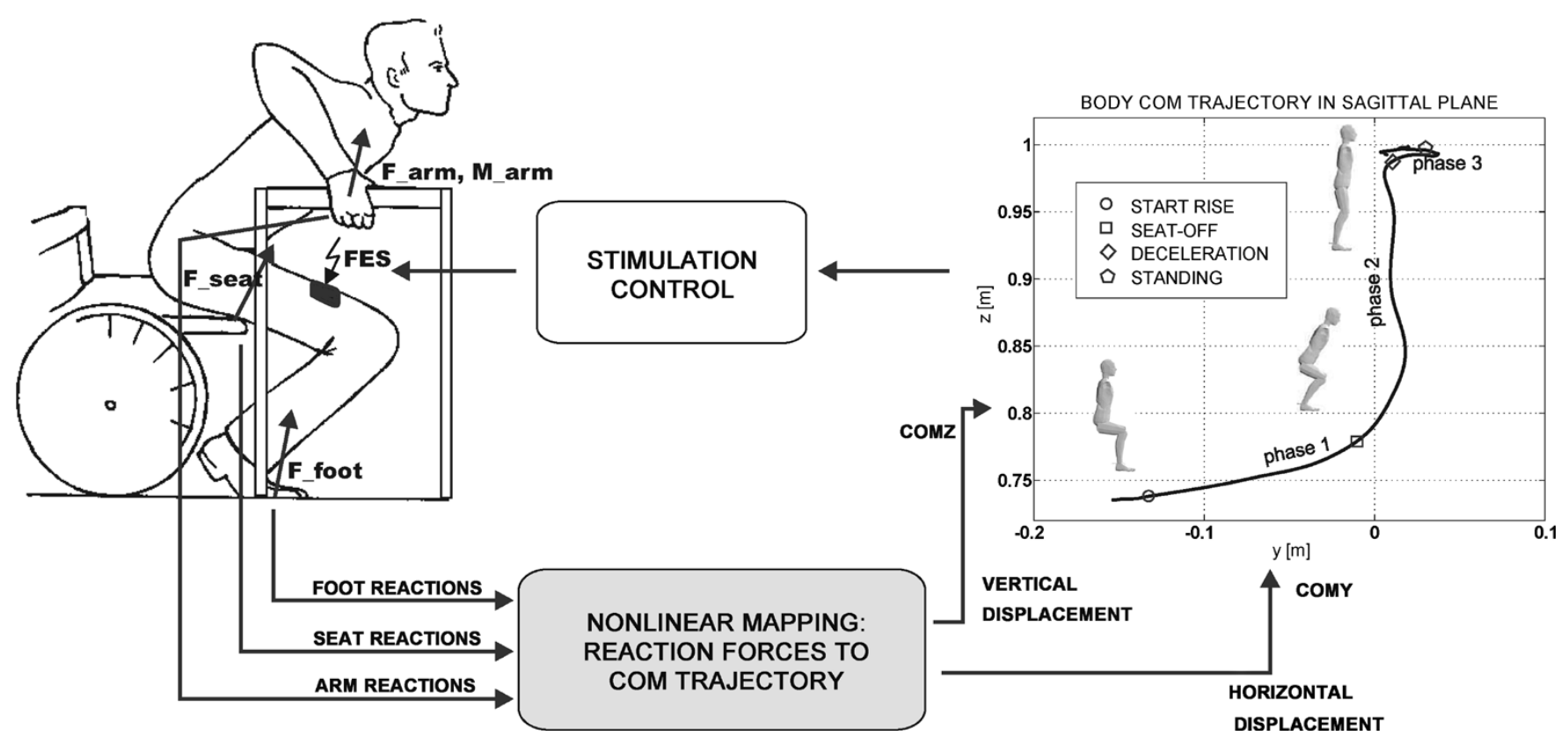

Fig. 1. Sensory-driven control concept in the FES-supported standing-up of paraplegic patient.

TABLE I

Data of Paraplegic Patients Participating In the StUdy

\begin{tabular}{c||c|c|c|c|l|c|c}
\hline $\begin{array}{c}\text { Patient } \\
\text { initials }\end{array}$ & Sex & $\begin{array}{c}\text { Age } \\
\text { [years] }\end{array}$ & $\begin{array}{c}\text { Height } \\
{[\mathrm{cm}]}\end{array}$ & $\begin{array}{c}\text { Weight } \\
{[\mathrm{kg}]}\end{array}$ & $\begin{array}{l}\text { Lesion } \\
\text { level }\end{array}$ & $\begin{array}{c}\text { Post injury } \\
\text { time [years] }\end{array}$ & $\begin{array}{c}\text { FES usage } \\
\text { [years] }\end{array}$ \\
\hline \hline AK & M & 44 & 180 & 74 & T10-11 & 1.5 & 0.5 \\
MK & M & 23 & 168 & 58 & T9 & 1.5 & 0.2 \\
SB & M & 31 & 183 & 64 & T10-12 & 1 & 0.9 \\
ZB & M & 22 & 184 & 94 & T3-4 & 3 & 2 \\
ZJ & F & 57 & 159 & 53 & T11 & 4.5 & 3 \\
BJ & M & 23 & 185 & 85 & T9 & 1.2 & 0.5 \\
MT & F & 28 & 171 & 75 & T4-5 & 7 & 5 \\
TM & F & 19 & 178 & 59 & T3-4 & 5 & 3.5 \\
\hline
\end{tabular}

sensors in robotics. An additional AMTI force plate was used for measuring the ground reaction forces under the right foot.

The motion kinematics of the body segments was assessed by the OPTOTRAK optical system (Northern Digital Inc., Waterloo, ON, Canada) measuring the three-dimensional (3-D) positions of active markers (infrared LEDs). Markers, about $1 \mathrm{~cm}$ in diameter, were attached to the body anatomical landmarks with doublesided tape. Two redundant sets of cameras were used in the measurements positioned $4 \mathrm{~m}$ right from the subject. The first set was positioned at a posterior angle of $45^{\circ}$ and the second at an anterior angle of $45^{\circ}$. With this setting, occlusions of markers caused by the walker frame or hand were minimized. Fig. 2 presents the standing-up manoeuvre of paraplegic patient performed in the measuring setup. Optotrak optical markers attached to the knee, elbow, and shoulder joints are well seen in Fig. 2.

2) Measurement Protocol: The subject was seated on the instrumented seat with the arms resting on the arm support frame. The height of the seat coincided with the height of a wheel chair, while the arm support frame height was adjusted according to the patient's preferences. Prior to measurements, three test standing-up trials were accomplished, followed by a certain amount of FES-assisted standing afterwards. This exercise enabled the subject to get used to the measuring equip-

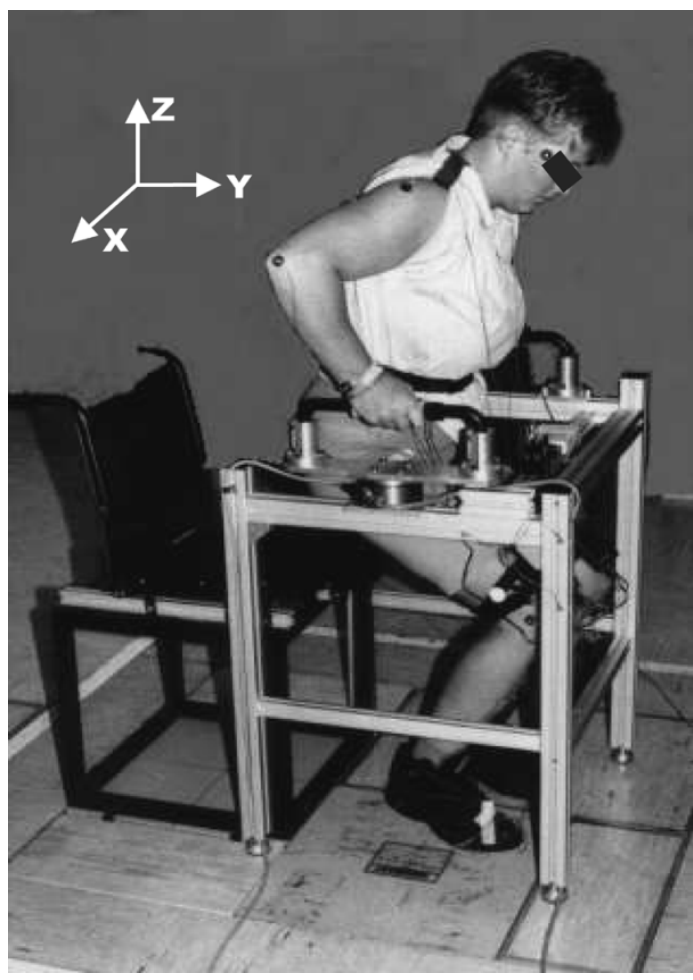

Fig. 2. Standing-up of paraplegic patient and a measurement setup.

ment and relieved the spasticity in paralyzed legs. No further consideration of spasticity effects was encountered since there was no significant evidence of spasticity during standing-up measurements in any of the subjects.

Surface stimulation of the M.quadriceps muscle group was used; they were stimulated open-loop with constant stimulation amplitude throughout the rising phase. The stimulation intensity was the level which brought the legs to their fully-extended position during sitting. The stimulation was voluntarily triggered on/off by the subject via the push-button mounted at the support 
handle. In measurement trials, the subject was asked to take the initial position and, after approximately $2 \mathrm{~s}$ from starting the data collection, he or she was asked to stand up in their preferred manner and speed. Five rising trials were recorded, with a 50-Hz sampling rate, for each participant, each measurement lasting for about $10 \mathrm{~s}$. By taking into consideration only five successive standing-up trials the results for each subject were consistent, because there was little muscle fatigue.

3) Measured Data Analysis: The signals were collected from active markers, force plates and a handle sensor. The signals were low-pass filtered using a fourth-order, dual-pass Butterworth filter with 5-Hz cutoff frequency. The positions of markers that had not been seen by cameras in short intervals were estimated by the cubic spline interpolation. The coordinate systems of all sensors were transformed to coincide with the reference coordinate system placed on the floor in the center of the support frame. The signal derivatives were calculated by differentiating the data followed by additional filtering. Using data, the motion of 3-D, 15 segment models of the subjects were calculated. The models embodied feet, shanks, thighs, pelvis, trunk, head, upper arms, lower arms, and hands. Each segment of the body had six degrees of freedom and was considered rigid. Each body joint was represented as a perfect ball-and-socket joint with no translation. From the marker positions, the joint center locations were determined and from them, the vectors along the segment longitudinal axes. Segmental masses and centers of mass locations were estimated using anthropometric relationships from the De Leva's study [25]. Masses were expressed as a percentages of total body mass, and the COM, lying on the segment's longitudinal axis, were estimated as percentage of the distance between proximal and distal joints. The total body COM location in each time instant was determined as a weighted sum of individual COM positions of all segments. The horizontal and vertical components of the body COM location in the sagittal plane were determined according to (1), where $m_{i}$ is the mass, while $y_{i}$ and $z_{i}$ are the horizontal and vertical displacements of particular segment

$$
\begin{aligned}
C O M_{Y} & =\frac{m_{1} \cdot y_{1}+m_{2} \cdot y_{2}+\ldots+m_{15} \cdot y_{15}}{m_{1}+m_{2}+\ldots+m_{15}} \\
C O M_{Z} & =\frac{m_{1} \cdot z_{1}+m_{2} \cdot z_{2}+\ldots+m_{15} \cdot z_{15}}{m_{1}+m_{2}+\ldots+m_{15}} .
\end{aligned}
$$

The accuracy of the COM assessment was verified by comparison with the COP determined from the measured support forces. The COP represents the point of application of the resultant of seat, arm, and foot ground reactions. In static conditions, the COP corresponds to the vertical projection of the body COM to the ground. The comparison was only possible in the horizontal plane. In this verification, all the measurement trials were analyzed considering the first 25 samples force record. Thus, the first half second of the measurement was analyzed when the subjects had been stationary in the initial position, waiting to be instructed to stand up. The resulting root mean square difference between horizontal COM and COP position among patients varied from $5.66 \pm 1.11 \mathrm{~mm}$ (subject AK) to $18.16 \pm 3.13$ $\mathrm{mm}$ (subject SB). The average difference was $8.08 \pm 4.11 \mathrm{~mm}$.
This result is less accurate than that reported for quiet standing, where the difference was around $1 \mathrm{~mm}$ [26], however, it shows that our method of COM estimation is relatively good. Compare this with Lenzi [27], who demonstrated that in the standing-up task the root-mean-square error (RMSE) in kinematics-based COM estimation can reach up to $38.3 \mathrm{~mm}$ when a $\pm 10 \%$ error in body segment parameters' estimation had been made.

In the final step of data processing, the body COM trajectory assessed in the inertial coordinates was transformed to the COM relative displacement according to the subject's initial position. This transformation enabled comparability of the measured data among subjects. Resulting trajectories of the lower extremity joints, the upper trunk inclination, and lower and upper body supportive forces are shown in Fig. 3 representing sample rising trials of eight paraplegic subjects. From the figure, it is evident that the duration of the sit-to-stand phase, rising speed, initial posture and the upper and lower extremity action varied considerably among the subjects.

Fig. 4 presents the COM displacements in sagittal plane with respect to the subject's initial position. Again, considerable variation in the approach to the sit-to-stand transfer can be observed among the subjects. Some of the patients transfer the upper body forward in the preparation phase and then rise vertically, while in others a dynamic horizontal transfer of the trunk occurs before the vertical lift.

From the measurements, three data sets were formed. For each of the subjects, the data from three standing-up trials was randomly assigned to two equal-sized sets; one the primary data set, for the model training procedure, and the other for validation during training. Measurements from the other two standing-up trials were designated test data set, and used in trained model evaluation.

\section{B. Input Variable Selection}

To be practicable for everyday usage, FES systems needs to employ as few physically-separate sensors as possible. Every feedback channel contributes to the complexity of the sensory device and to the wiring and mounting difficulties. Therefore, the question: what feedback information is just sufficient for recognition of the body state - in our case, the body COM trajectory - is crucial. We investigated the minimal requirements for feedback information. We divided the potential feedback sources into ten groups, each group incorporating different numbers of variables. The groups were formed so that we could examine the functionality, practicality and feasibility of various configurations of sensors.

The empirical input variable groups are listed in Table II. Group 1 encompasses all the possible signals acquired in the measurement setup, i.e., arm, seat and foot reactions. Additionally the derivatives of signals were incorporated to verify their information significance to the output: we expected better modeling of dynamic movements. In the case of ground reactions, beside the three components of the reaction force, the position of foot center of pressure was used. The position is expressed in the coordinates of the foot sole and normalized to the foot length. The components are denoted as $c o p_{x}$ and $c o p_{y}$. The seat reaction force, assessed by the force plate, is a 3-D vector, while 

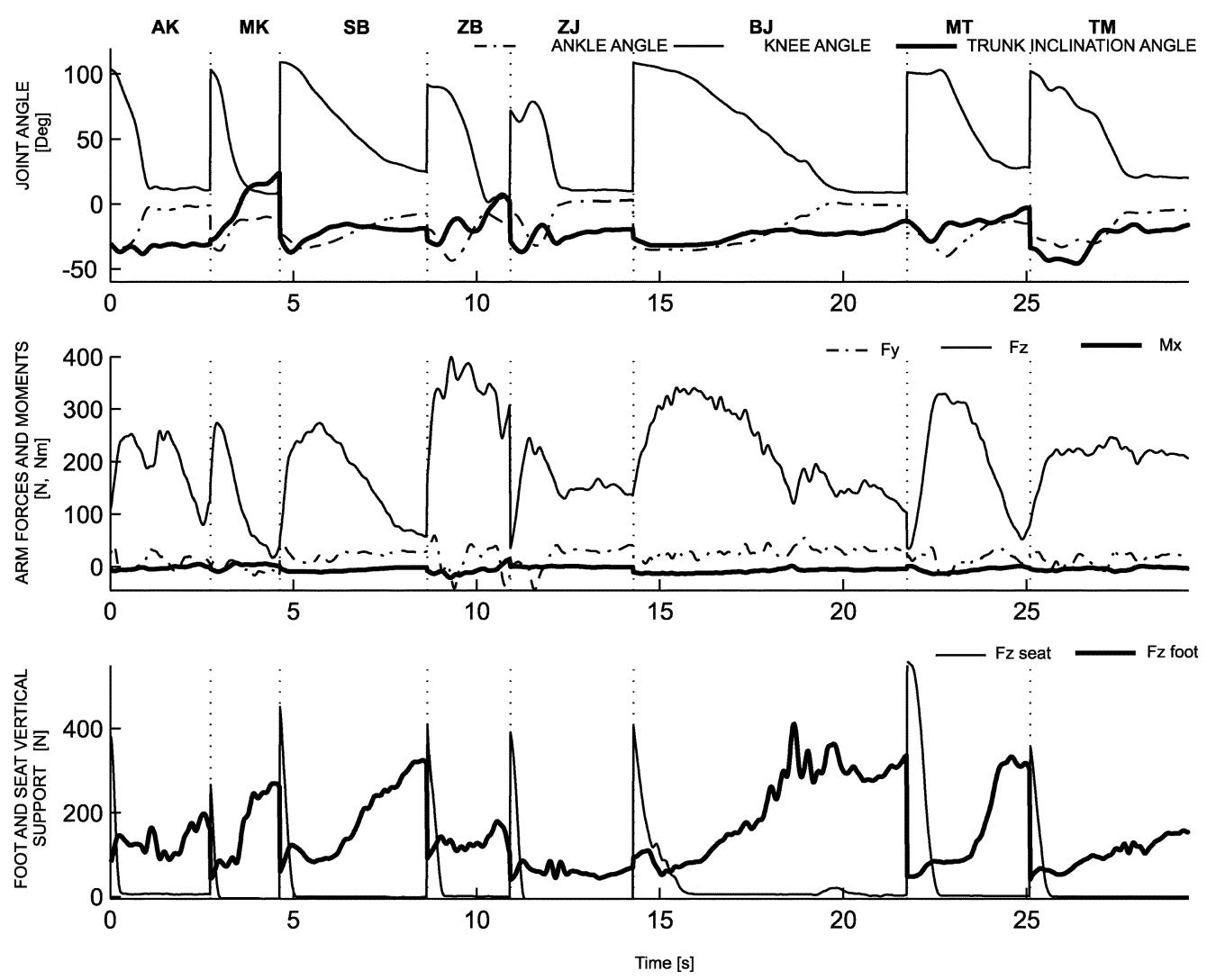

Fig. 3. Motion trajectories and supporting actions of the upper and lower extremities in sample trials of eight paraplegic subjects. The portion of the data between the dotted lines belongs to one sample standing-up of one subject.

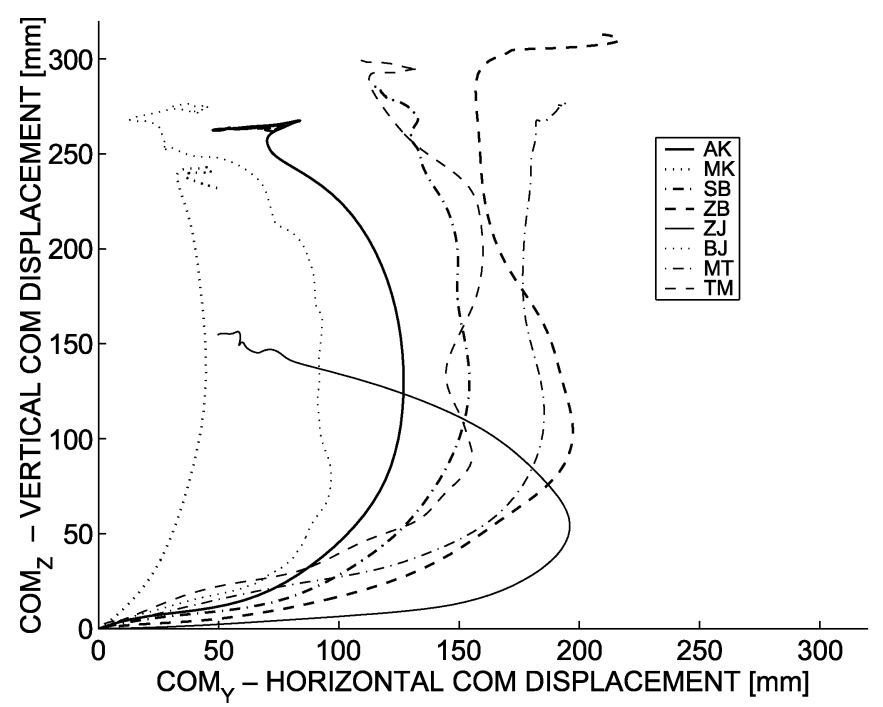

Fig. 4. Sample COM displacements in sagittal plane for eight paraplegic subjects.

the arm reactions, when assessed by the JR3 force sensor, consists of three force and three moment components. For comparison, Group 2 excludes the derivatives of the signals. Group 3 excludes the seat reaction force signals since sensors attached to the seat or wheelchair are less practicable. Group 4 incorporates only the vertical component of the foot reactions, since this is the variable when shoe insole sensors can be used instead of the force plate. Group 5 investigates the usage of simpler and less expensive sensors for measuring arm support forces.
Only vertical and horizontal handle reaction components were used in this case in combination with the shoe insole sensory signals. Group 6 investigates the use of only the shoe insole sensor. Additionally, the possible combinations of the shoe insole sensor with the goniometers, inclinometer or accelerometers were investigated. Thus, the ankle joint angle was incorporated in Group 7 and the knee joint angle in Group 8. Group 9 verifies the combination with inclinometer mounted at the upper body, while Group 10 verifies the shoe insole combination with the accelerometers attached to the trunk.

The significance of each group was evaluated using a modeling approach. Two different nonlinear models were used to predict the body COM trajectory from the input signals of each group. The RMSE between the actual COM trajectory and the model-predicted output were calculated to assess the model performance for each group. RMSE values were calculated separately for the horizontal and vertical component of the COM trajectory as

$$
\begin{aligned}
\operatorname{RMSE}_{Y} & =\sqrt{\frac{1}{N} \sum_{k=1}^{N}\left(C O M_{Y k}^{m}-C O M_{Y k}^{a}\right)^{2}} \\
\operatorname{RMSE}_{Z} & =\sqrt{\frac{1}{N} \sum_{k=1}^{N}\left(C O M_{Z k}^{m}-C O M_{Z k}^{a}\right)^{2}}
\end{aligned}
$$

where superscript $a$ stands for actual and $m$ for modeled value of the COM trajectory in a sample $k$. In (2), parameter $N$ represents a number of data points in particular test data set. 
TABLE II

FEEDBACK SIGNALS IN TEN INPUT GROUPS

\begin{tabular}{|c|c|}
\hline Group 1 & $\begin{array}{l}\text { FOOT }\left(\operatorname{cop}_{x}, \operatorname{cop}_{y}, F_{x}, F_{y}, F_{z}, \dot{F}_{x}, \dot{F}_{y}, \dot{F}_{z}\right) \\
\text { SEAT }\left(F_{x}, F_{y}, F_{z}, \dot{F}_{x}, \dot{F}_{y}, \dot{F}_{z}\right) \\
\text { ARM }\left(F_{x}, F_{y}, F_{z}, M_{x}, M_{y}, M_{z}, \dot{F}_{x}, \dot{F}_{y}, \dot{F}_{z}, \dot{M}_{x}, \dot{M}_{y}, \dot{M}_{z}\right)\end{array}$ \\
\hline Group 2 & $\begin{array}{l}\text { FOOT }\left(\operatorname{cop}_{x}, \operatorname{cop}_{y}, F_{x}, F_{y}, F_{z}\right) \text {, } \\
\text { SEAT }\left(F_{x}, F_{y}, F_{z}\right) \\
\text { ARM }\left(F_{x}, F_{y}, F_{z}, M_{x}, M_{y}, M_{z}\right)\end{array}$ \\
\hline Group 3 & $\begin{array}{l}\text { FOOT }\left(\operatorname{cop}_{x}, \operatorname{cop}_{y}, F_{x}, F_{y}, F_{z}, \dot{F}_{x}, \dot{F}_{y}, \dot{F}_{z}\right) \\
\text { ARM }\left(F_{x}, F_{y}, F_{z}, M_{x}, M_{y}, M_{z}, \dot{F}_{x}, \dot{F}_{y}, \dot{F}_{z}, \dot{M}_{x}, \dot{M}_{y}, \dot{M}_{z}\right)\end{array}$ \\
\hline Group 4 & $\begin{array}{l}\text { FOOT }\left(\operatorname{cop}_{x}, \operatorname{cop}_{y}, F_{z}, \dot{F}_{z}\right) \\
\text { ARM }\left(F_{x}, F_{y}, F_{z}, M_{x}, M_{y}, M_{z}, \dot{F}_{x}, \dot{F}_{y}, \dot{F}_{z}, \dot{M}_{x}, \dot{M}_{y}, \dot{M}_{z}\right)\end{array}$ \\
\hline Group 5 & $\begin{array}{l}\text { FOOT }\left(\operatorname{cop}_{x}, \operatorname{cop}_{y}, F_{z}, \dot{F}_{z}\right) \\
\text { ARM }\left(F_{y}, F_{z}, \dot{F}_{y}, \dot{F}_{z}\right)\end{array}$ \\
\hline Group 6 & FOOT $\left(c o p_{x}, c o p_{y}, F_{z}, \dot{F}_{z}\right)$ \\
\hline Group 7 & $\begin{array}{l}\text { FOOT }\left(\operatorname{cop}_{x}, \operatorname{cop}_{y}, F_{z}, \dot{F}_{z}\right) \\
\text { ANKLE JOINT ANGLE }\left(\phi_{\text {ankle }}, \dot{\phi}_{\text {ankle }}\right)\end{array}$ \\
\hline Group 8 & $\begin{array}{l}\text { FOOT }\left(\operatorname{cop}_{x}, \operatorname{cop}_{y}, F_{z}, \dot{F}_{z}\right) \\
\text { KNEE JOINT ANGLE }\left(\phi_{\text {knee }}, \dot{\phi}_{\text {knee }}\right)\end{array}$ \\
\hline Group 9 & $\begin{array}{l}\text { FOOT }\left(\operatorname{cop}_{x}, \operatorname{cop}_{y}, F_{z}, \dot{F}_{z}\right) \\
\text { TRUNK INCLINATION ANGLE }\left(\phi_{\text {trunk }}, \dot{\phi}_{\text {trunk }}\right)\end{array}$ \\
\hline Group 10 & $\begin{array}{l}\text { FOOT }\left(c o p_{x}, \operatorname{cop}_{y}, F_{z}, \dot{F}_{z}\right) \\
\text { TRUNK ACCELERATION }\left(a_{y}, a_{z}\right)\end{array}$ \\
\hline
\end{tabular}

\section{Artificial Neural Network (ANN) Model}

As an example of a well-established approach, a multilayer perceptron ANN was trained and the network performance characteristics examined [28]. The size of the network was kept constant to make sure that the comparison is valid. The neural network was built in the Mathworks Matlab software environment as a two-layer feedforward network. The first layer incorporated six neurons with a hyperbolic tangent sigmoid activation function, while the second layer consisted of two neurons with a linear activation function. The back-propagation learning algorithm, based on Levenberg-Marquardt optimization was used for training the network. In training, the error cost on the validation set was used to stop training early if further training on the primary training set would hurt generalization to the validation set. The network was trained for up to 300 epochs to an error tolerance of $10^{-5}$. Performance on the third, test set was then used to measure how well the network generalizes beyond primary and validation sets.

\section{Gaussian Process (GP) Prior for Regression and Hierarchical Mixture Models}

As an alternative to neural networks, we also used a GP prior regression model. An introduction to this approach is given in reviews by [29] and [30]. The key equations are included in the following.

Given $N$ data points of training data $\left\{y_{n}, x_{n}, n=1, \cdots, N\right\}$, where $x$ is a $Q$-dimensional vector of inputs, and $y$ is the output. A Gaussian process is defined in such a way that $y(x)$ has a Gaussian prior distribution with zero mean and covariance function $C\left(x_{i}, x_{j}\right)=\operatorname{Cov}\left(Y\left(x_{i}\right), Y\left(x_{j}\right)\right)$. An example of such a covariance function is

$$
\begin{aligned}
C\left(x_{i}, x_{j}\right)= & C\left(x_{i}, x_{j} ; \theta\right) \\
= & v_{0} \exp \left(-\frac{1}{2} \sum_{q=1}^{Q} w_{q}\left(x_{i q}-x_{j q}\right)^{2}\right)+a_{0} \\
& +a_{1} \sum_{q=1}^{Q} x_{i q} x_{j q}+\delta_{i j} \sigma_{v}^{2},
\end{aligned}
$$

where $\theta=\left(w_{1}, \cdots, w_{Q}, v_{0}, a_{0}, a_{1}, \sigma_{v}^{2}\right)$, and $\delta_{i j}=1$ if $i=j$ and 0 otherwise. This covariance function is often used in practice [32] - it has several terms which describe the variability in the data. The first term $v_{0} \exp \left(-(1 / 2) \sum_{q=1}^{Q} w_{q}\left(x_{i q}-x_{j q}\right)^{2}\right)$ indicates that points close together are more correlated than points far apart-a smoothness assumption, where the parameter $w_{q}$ reflects the lengthscale parameter for the $q$ th input. The smaller $w_{q}$, the less the output changes as $x_{q}$ is changed. The term $a_{1} \sum_{q=1}^{Q} x_{i q} x_{j q}$ is the covariance associated with a linear model - a practical assumption. The term $\delta_{i j} \sigma_{v}^{2}$ is associated with measurement noise, representing noise of variance $\sigma_{v}^{2}$ on the observations. More discussion about the choice of covariance function and the details of the implementation of the model can be found in [30]. The parameters $\theta$ of the covariance function can be optimized by maximizing the log-likelihood

$$
L(\theta)=-\frac{N}{2} \log (2 \pi)+\frac{1}{2} \log \Sigma+\frac{1}{2} \mathbf{y}^{t} \Sigma \mathbf{y}
$$

using standard optimization techniques such as conjugate-gradient approaches, or by integrating over them using numerical methods such as Markov-Chain Monte Carlo methods.

We form a covariance matrix $\Sigma$, the elements of which are $\sum_{i j}=C\left(x_{i}, x_{j} ; \theta\right)$, and we can say that the probability of the observed data is

$$
p\left(y_{1}, \ldots, y_{N} \mid x_{1}, \ldots, x_{N}\right)=\mathcal{N}(0, \Sigma) .
$$

To find the conditional distribution for the model, conditioned on $\mathbf{x}, \mathbf{y}$, and a new test input $x^{*}$, we have a mean and variance of

$$
\begin{aligned}
\mu & =\mathbf{k}\left(x_{*}\right) \Sigma^{-1} \mathbf{y} \\
\sigma^{2} & =k\left(x_{*}\right)-\mathbf{k}\left(x_{*}\right)^{T} \Sigma^{-1} \mathbf{k}\left(x_{*}\right)
\end{aligned}
$$

where $\mathbf{k}\left(x_{*}\right)$ is the vector of covariances between the output at $x_{*}$ and the training data, i.e., $\mathbf{k}_{i}\left(x_{*}\right)=C\left(x_{*}, x_{i}\right)$. An alternative expression for (6) is to use

$$
\mu=\boldsymbol{\alpha}^{T} \mathbf{y} \quad \text { with } \quad \boldsymbol{\alpha}^{T}=\mathbf{k}\left(x_{*}\right) \Sigma^{-1}
$$

which emphasizes that the mean predictions are a linear smooth of the training data, where that smoothing function is dependent on the current input $x_{*}$. 

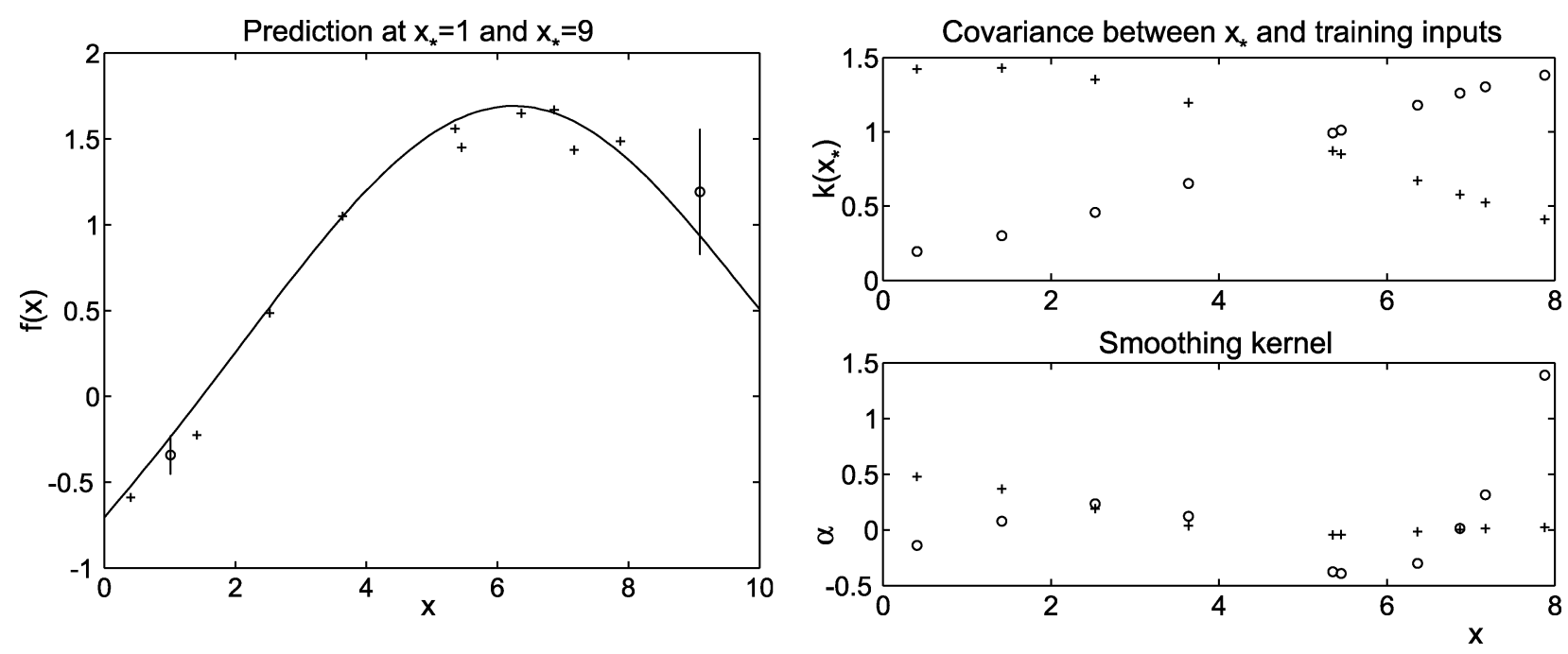

Fig. 5. (Left) GP modeling of the noisy observations (crosses). Mean predictions at $x_{*}=1$ and $x_{*}=9$ (circles) with their associated $\pm 2 \sigma$ error-bars, along with the true function (continuous line). (Right) covariances between the test and the training inputs (upper plots), and smoothing kernels $\boldsymbol{\alpha}$ (bottom plots). The crosses correspond to $x_{*}=1$ and the circles to $x_{*}=9$.

1) Illustrative Examples of the Use of GP Models: The following example ${ }^{1}$ illustrates the GP modeling of noisy data. In this particular case, we choose the data to actually come from a realization of a zero-mean Gaussian Process with Gaussian covariance function, where $w=0.04$ (corresponding to a correlation length of 5) and $v_{0}=2$, given a one-dimensional argument $x$ in $[0,10]$. We select $N=10$ training cases at random and corrupt the outputs with a white noise with variance 0.01 . Starting the optimization of the minus log-likelihood with an initial estimate of 1 for all parameters, it converges to $L(\theta)=-0.9765$ after 100 iterations. The ML parameters found are $w=0.0531$, $v_{0}=1.4363$, and $\sigma_{v}^{2}=0.0076$. Both $v_{0}$ and $\sigma_{v}^{2}$ are underestimated, whereas $w$ is overestimated, but these values are satisfactory, considering the very few number of data points and their unevenly spread in the input space. We then make two predictions, at $x_{*}=1$ and $x_{*}=9$. Fig. 5 (left) shows the underlying function (that is the realization of the 'true' underlying GP), the training cases (crosses) and the predictions (circles) with their $\pm 2 \sigma$ error-bars. The upper-right plot corresponds to the covariances between the test and training cases (for $x_{*}=1$, crosses and $x_{*}=9$, circles) and the bottom-right plot to the smoothing kernels. For $x_{*}=1$, which is between training points, the predictive variance is small, as the model is confident about its prediction but for $x_{*}=9$, the error-bars are significantly larger, as the test input lies in a region where there are few or no training inputs. Indeed, the plot of the covariances between the test and training inputs indicates that, for $x_{*}=9$, the covariances diminish more rapidly and to smaller values than those with $x_{*}=1$.

Fig. 6 shows samples drawn from the zero-mean GP prior (dashed lines) and from the predictive-posterior process, conditioned on the training data and 100 test inputs $x_{*}$ in $[0,10]$ (that is, the realizations are drawn from a 100-dimensional normal distribution, with mean vector $\mu\left(x_{*}\right)$ and covariance matrix $\sigma^{2}\left(x_{*}\right)$, computed for 100 values of $x_{*}$. Notice the "edge effect" from $x=8$, where no more training points are present.

${ }^{1}$ Many thanks to A. Girard for providing this example and figures.

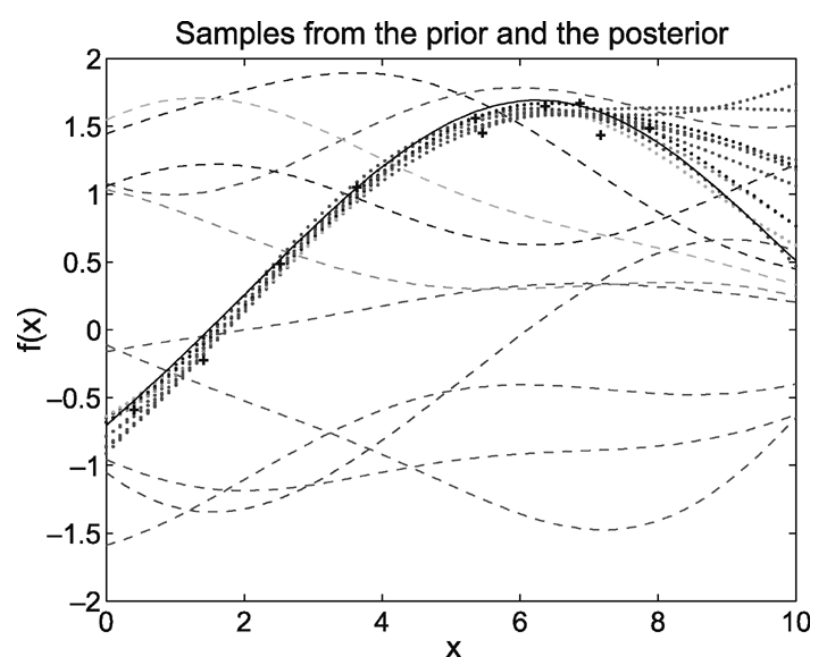

Fig. 6. Samples from the prior process (dashed lines) and from the posterior (dotted lines), conditioned on the training data (crosses), for $x \in[0,10]$.

2) Example of GPs in Use for the Standing-Up Data: To illustrate the prediction of uncertainty provided by GP models, we use an example of prediction of $C O M_{x}$ and $C O M_{y}$ for five separate standing-up trajectories of patient BJ. Fig. 7 shows the mean and \pm 2 standard deviation uncertainty bands from a single GP. The GP included some data from each of the first three trajectories in the training set, and the second two were test data. Note that the uncertainty is low on the predictions on data close to the training data, but increases for the data points further from the training data. The uncertainty also varies within individual batches, depending on the input state, reflecting variations in model complexity, and training data density.

3) Comparison With Neural Networks: An empirical comparison in [32] showed that GPs were usually as good as or better than neural networks in test comparisons. GPs tend to have a clearer advantage in problems with smaller data sets. The major difference is that the training data are retained by the model and predictions are inferred from those data, rather than the parametric approach of neural networks, where the data points are 

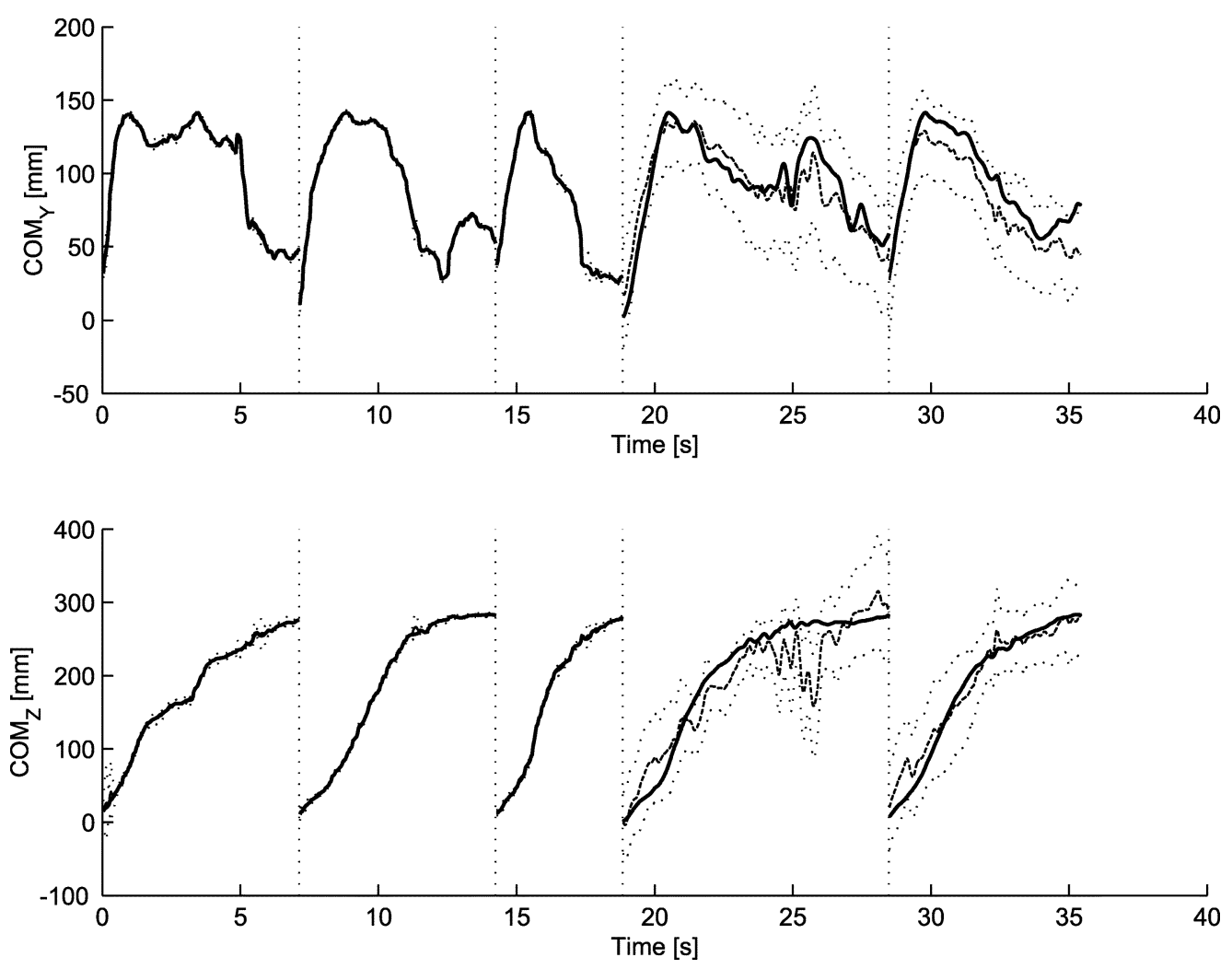

Fig. 7. Example of GP prediction, showing mean and two standard deviations for five separate standing-up trajectories of patient BJ. The $x$ axis indicates time. The first three sets were included in the training set, and the second two were test data. Actual COM trajectory is represented by bold solid line, the GP model response is represented by dashed line and its $95 \%$ confidence interval is represented by dotted line.

represented by a finite number of parameters, and discarded. This means that prediction uncertainty in GPs can be made to increase as we make predictions further from the training data (in terms of the input space), but it also has storage and computational issues, compared to neural networks, as training set sizes increase. It also means that models can include new data points relatively easily, without major retraining.

4) GPs and Input Subset Selection: GPs allow a "soft model-structure selection," where the complexity of the model, as measured by the effective degrees of freedom [33] can vary automatically with the hyperparameters. It also provides an automatic relevance detection, as the length-scale parameters $w_{q}$ associated with input $q$ give an indication of how important any given input is - if an element of input vector does not help predict outputs accurately, the $w_{q}$ will tend to go toward zero, as likelihood is maximized [30].

5) Hierarchical GP Regression Model: The implementation of a GP regression model requires the inversion of a covariance matrix, the dimension of which is the sample size of the training data. It becomes computationally expensive for large sample sizes $(N>1000)$, because the computational cost scales as $O\left(N^{3}\right)$. For the data discussed in this paper, if we consider a single standing-up, a single GP regression model is not computationally problematic. However, if we want to combine the data collected from the different standings-up and from the different patients, the sample size may be as large as a few thousand data points, and the use of a hierarchical mixture model, as proposed in [31] is recommended. This model also allows for the heterogeneity for the data-set combining from the different sources, which is a particularly nice property for data acquired in human motion, as is the case in our study.

The proposed hierarchical GP regression model has the following two-level structure: a lower-level single GP regression model defined around (4) is used to fit the data corresponding to each replication (different standing-up) separately, and the structures of the basic models are similar but with some mutual heterogeneity; a higher-level model is defined to model the heterogeneity among different replications. Specifically, suppose that there are $M$ different replications. In the $m$ th group, $N_{m}$ observations are collected. Let the observation be $y_{m n}$, $m=1, \cdots, M, n=1, \cdots, N_{m}$. In a hierarchical mixture model of Gaussian processes for regression, we have

$$
y_{m n} \mid z_{m}=k \sim G P\left(\theta_{k}\right)
$$

where $z_{m}$ is an unobservable latent indicator variable. If $z_{m}=$ $k$ is given, the model for group $m$ is a GP regression model $G P\left(\theta_{k}\right)$, as defined around (4). The association among the different groups is introduced by the latent variable $z_{m}$, for which

$$
P\left(z_{m}=k\right)=\pi_{k}, \quad k=1, \ldots, K
$$

for each $m . K$ is the number of components of the mixture model. We assume that $K$ has a fixed value. For the details of the theory and implementation refer to [31].

\section{MODELING RESULTS}

In the following section, the prediction results from the nonlinear models are presented. The performances of the proposed 
ACTUAL COM TRAJECTORY (-) AND ANN MODEL RESPONSE $(\longrightarrow)$ HORIZONTAL COM DISPLACEMENT - COMY

VERTICAL COM DISPLACEMENT - COMZ
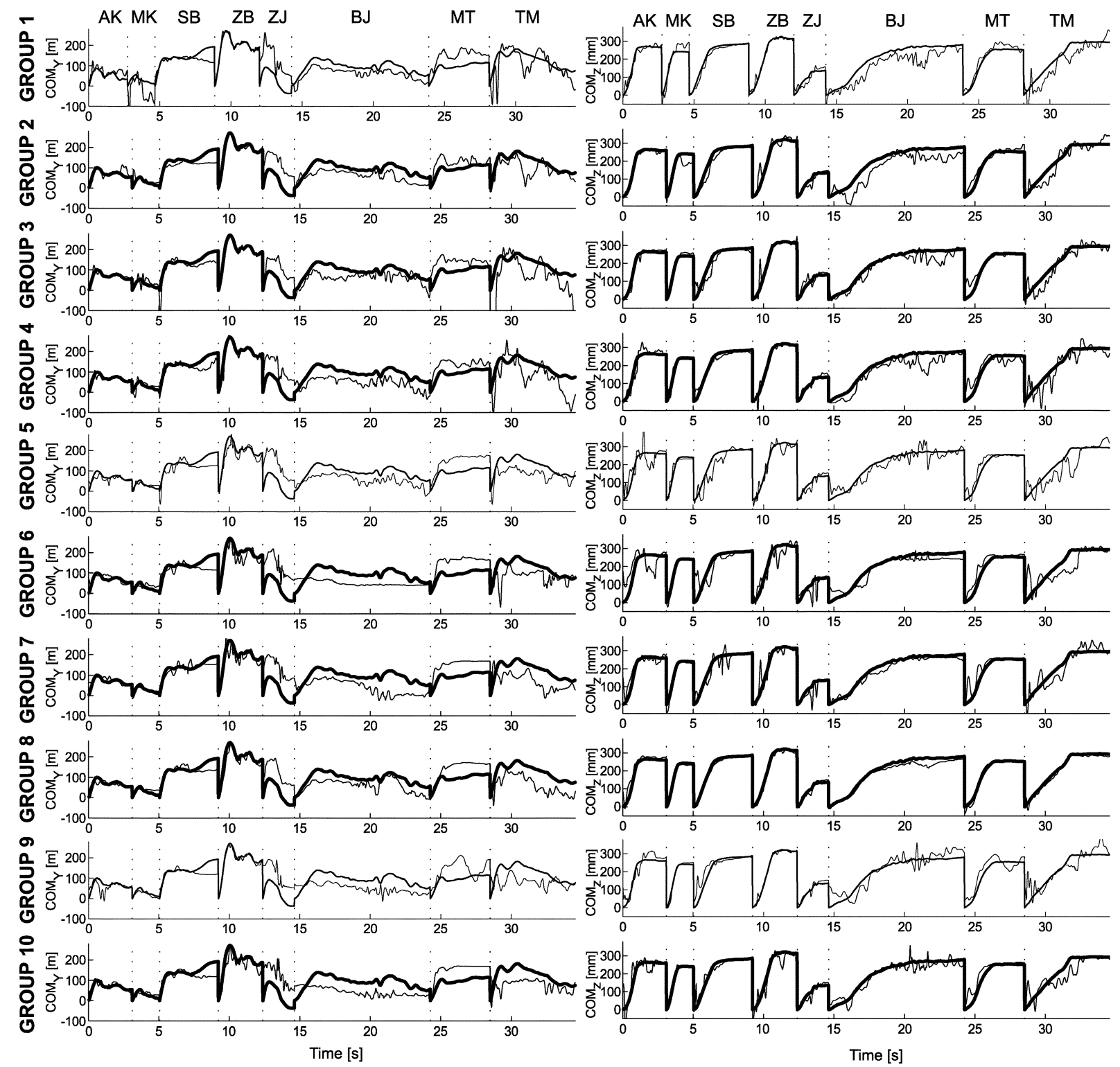

Fig. 8. Comparison of resulting ANN model predictions and the COM displacements measured in the fourth (testing) trial of real standing-up for eight paraplegic subjects and ten different groups of input variables.

ANN and GP regression models are verified on prediction of the body COM position. For each subject and for each input group, an individual model was built and verified with the subject's test data set. The model structure depended on the specific subset sensors used to provide the input vector. The input variables were organized as described in Section II-B.

\section{A. Model Predictions Compared to Test Data}

Figs. 8 and 9 compare the resulting ANN and GP model predictions with the COM displacements measured in the fourth (testing) trial of real standing-up. In the figures, the results for the horizontal and vertical component of the COM trajectory are shown separately in the left and right column, respectively. Each graph in the figure is divided into eight sections, successively demonstrating the results for eight subjects who participated in the study. The sections are annotated with the subject's initials on top of the figure. For example, the first section in the second row in the right of Fig. 8 compares the ANN model output with the real COM vertical displacement in the fourth standing-up of subject AK when utilizing the model input variables from Group 2. In Figs. 8 and 9, the deterioration of the model performance as a consequence of decreasing the number of model input channels can be observed.

\section{B. Relative Importance of Input Signal Groups}

To get a better insight into the significance of particular groups of input signals to the model output, all the test RMSE values of all the subjects were averaged and compared in two bar graphs. The RMSE values between the modeled and actual COM trajectories were calculated according to (2). Other 


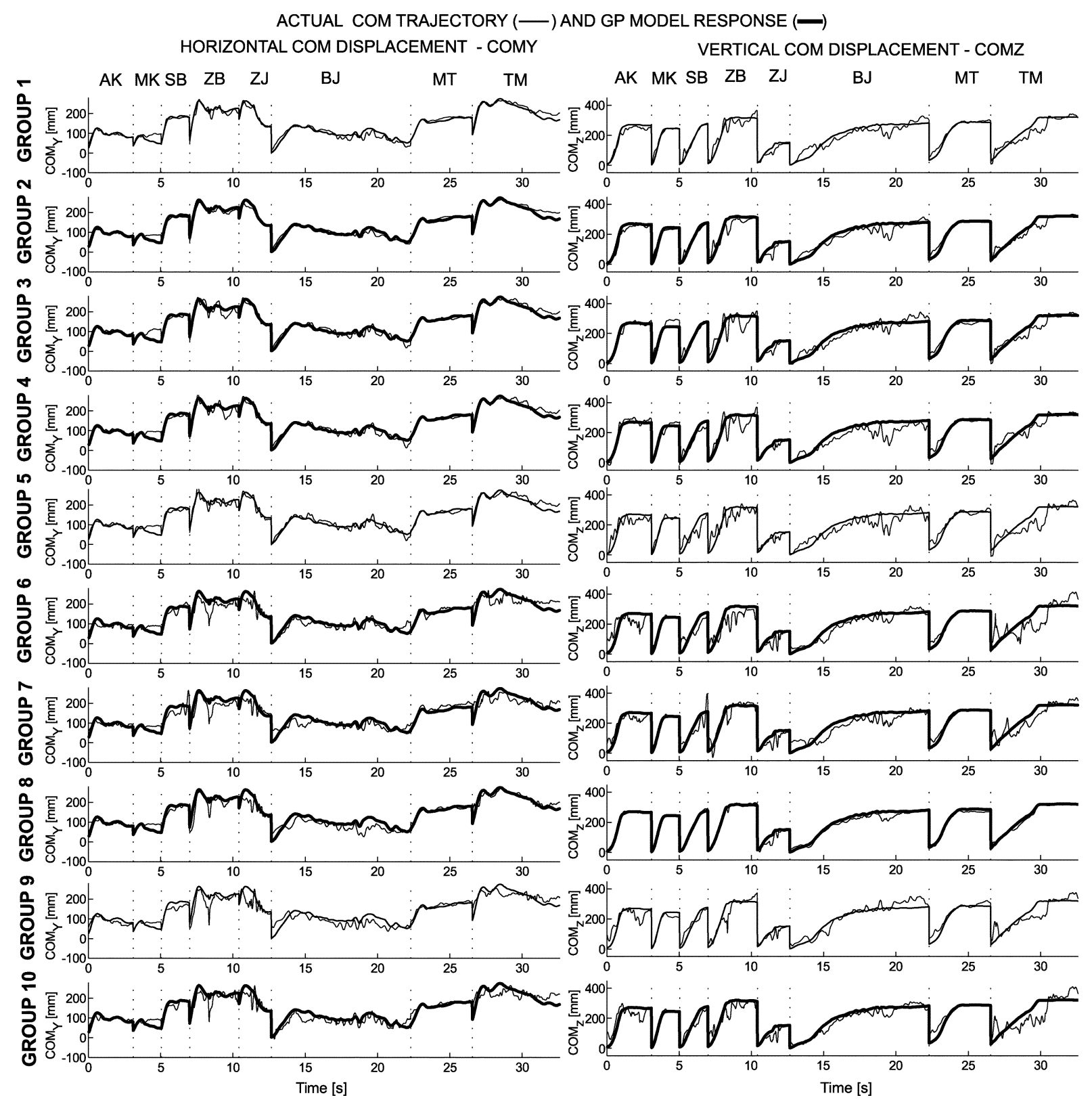

Fig. 9. Comparison of resulting GP model predictions and the COM displacements measured in the fourth (testing) trial of real standing-up for eight paraplegic subjects and ten different groups of input variables.

model evaluation measures such as the $95 \%$ confidence interval or correlation produced similar results. Therefore, only the RMSE was used for evaluation of the models. The comparison bar graphs, presented in Fig. 10, illustrate the averaged ANN model results on the left and the averaged GP model results on the right. Again, the results are presented separately for the horizontal and vertical component of the COM trajectory.

\section{DISCUSSION}

From Figs. 8-10, we can see that both approaches give quite good results, although GP modeling seems to provide a more accurate model. An example of one of these subplots with $2 \sigma$ uncertainty bounds was given in Fig. 7. The bar graphs confirm our assumptions about the information importance of the input groups. The degradation of the model performances with respect to the number of input channels can be noticed. It is interesting that the pattern of variability among subjects is not similar in the ANN and GP results. For example, the worst results for COMY in ANN modeling were achieved with the subject ZJ who was standing-up, according to Fig. 4, with the extensive forward excursion before rising. On the other hand, the worst results in GP modeling were achieved with the subject MK who was standing-up in a primarily vertical manner. The GP tends to be worse in the vertical rather than the horizontal component, which may be because of a zero-mean assumption in the standardization used. This seems well-suited to the horizontal component, but more information about the patient, such as height, for example, is needed to improve on the vertical component.

The overall modeling results presented in Fig. 10 illustrate the information significance of input groups defined in Table II. 

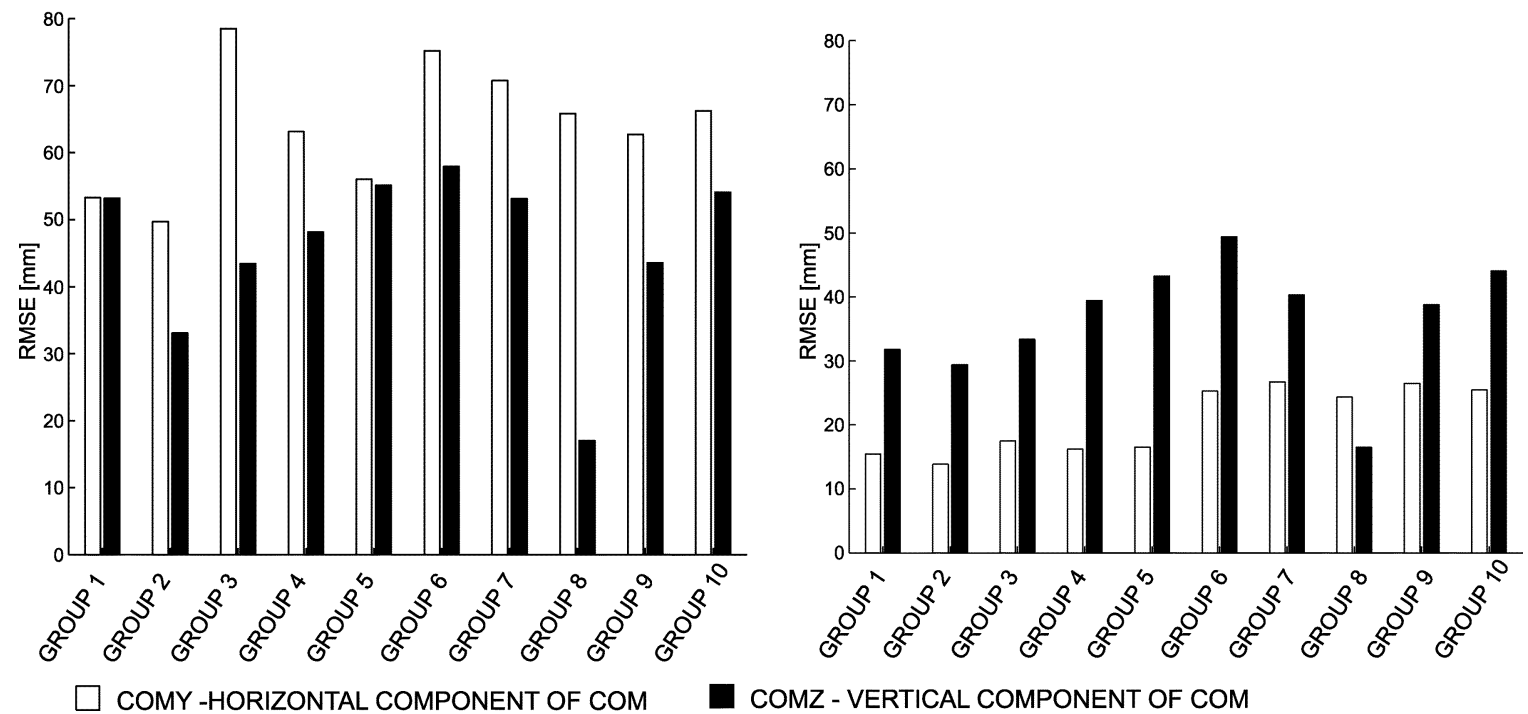

Fig. 10. Overall modeling results for each group of inputs, summed over all patients.

According to the data from literature and the verification results from static condition, the RMSE between 10 and $20 \mathrm{~mm}$ is considered satisfactory result for the COM assessment in antero/posterior direction. Such a result would qualify the sensory system for a balance control task also. For the COM assessment in vertical direction, there is no study or data available for comparison. From the bar graphs it can be observed that the input information about the knee joint motion (see Group 8) improves the model performance significantly. Obviously, the knee joint angle is closely correlated with the body vertical position. Taking the RMSE result of the Group 8 for a reference, the double error range, i.e., RMSE up to $37 \mathrm{~mm}$, is considered an acceptable accuracy for the model.

Observing the results for each particular input group, it is first interesting that the models exhibit better performance when the signal derivatives are excluded from the input (compare the results of Groups 1 and 2). This phenomenon can be attributed to the numerical differentiation of noisy force signals. On this basis, we can conclude that the incorporation of the derivatives of forces is not beneficial for the model performance.

Secondly, it can be seen that the peak of RMSE values is attained when only the instrumented foot insole information is used for the feedback in Group 6. Utilization of this sensor alone apparently does not meet the desired accuracy criteria.

Next, it is interesting to compare the results between Groups 1, 2, and 3, and Groups 4 and 5. The former are based on the sensory signals that are difficult to acquire in a practical manner with today's technology, while the latter incorporate practical and easy implementable sensors. Excluding the sensory channels improves the practicality but deteriorates the performance of models. However, notice that the sensory system based on the GP modeling and the signals from Group 4 and conditionally also Group 5 would meet the accuracy requirements.

Finally, the averaged results demonstrate that in the conditions under which the data was acquired the utilization of in- formation about force reactions at the input is a comparable alternative to the information about the ankle joint angle, trunk inclination angle, and trunk acceleration.

\section{CONCLUSION}

We have analyzed the information available from sensors when paraplegic patients stand up. The analysis focused on the use of the support force signals for the purpose of body state estimation. The body COM trajectory has been estimated utilizing two different nonlinear modeling approaches. The results of the study proved that a force-feedback-based FES system is viable and realistic. Regardless of the fact that the study was accomplished with data acquired in a laboratory with sophisticated measurement equipment, conclusions can be drawn for practical portable systems. We searched for the minimal number and complexity by comparing different sets of signals. In the study, the accuracy criteria for the COM assessment were assigned having in mind that the balancing task in FES-supported standing-up in paraplegia is performed by the subject. The balance control is, therefore, not the objective task for the FES system allows lower required accuracy. It was also beyond the scope of the study to search for the optimal feedback set for a particular sensor-supported FES system. Nevertheless, the results suggest a feasible and satisfactory configuration. They show that both the foot and arm reactions are vital for the COM trajectory reconstruction, but the seat force sensor is unneccesary. Inclusion of the derivatives of forces was not beneficial since it led to a deterioration in modeling performance. It can, therefore, be concluded that the combination of sensors from Group 4, i.e., the multidimensional force sensor used for handle reactions and the foot insole for the vertical foot reaction and its COP, would provide sufficient accuracy and would be also practicable with currently-available technology. For the future, we may expect progress in the development of wearable multidimensional force sensors for ground reaction forces. In that case, the Group 3 arrangement could be implemented. As a 
side comparison, we investigated the significance of kinematic parameters to the COM trajectory reconstruction and showed that information about knee joint angle is most descriptive. Based on this cognition we may speculate, since the example was not verified in the study, that the combination of the handle and foot force sensors from Group 4 and the knee goniometer from Group 8 would form a highly accurate system for the vertical and horizontal COM assessment. However, increasing the assessment accuracy on one side, would on the other side decrease the practicality of the system requiring the attachment of goniometers.

On one hand, the study provided knowledge of the significance of the various feedback signals and will thus ease the design of sensory supported FES systems. On the other hand, the study is practical comparison between the ANN and GP nonlinear modeling methods. The modeling performance shows that although GPs are computationally more expensive, they provided a better fit to the data. They also have the advantage that they provide an estimate of the conditional density for predictions, rather than just the conditional mean, as provided by the neural network. This is compatible with earlier empirical comparisons between GP's and neural networks in [32]. GP's tend to perform better than neural nets in cases where training data is sparse, while neural nets can be better when there is a large amount of training data, and the function to be learned is of varying complexity (which would require a nonstationary covariance function for the GP).

The hierarchical GP was computationally much more efficient than a single GP, and also coped well with the heterogeneity among patients. Since we observed great variability in standing-up among paraplegic subjects (subjects differed in sex, age, weight, height, and the level of spinal-cord injury, while data even varied in the same subject due to variance in initial position and muscle fatigue), results suggest that the models used in this paper should be further calibrated to an individual subject.

In terms of computational requirements, the neural network has a very small memory footprint, requiring storage only of the network weights which is the product of the number of inputs $x$ number of hidden units $\times$ number of outputs, while the GP might be storing several thousand training examples, and inference to new points involves multiplication of the inverse covariance matrix (which can be calculated off-line, prior to use), by the covariance with the test point, which for a single test point would involve $N^{2}+N$ floating-point multiplication and addition operations for $N$ training points. For the hierarchical model, we have $\sum_{i}^{M}\left(N_{i}^{2}+N_{i}\right)$, operations where $N_{i}$ are the sizes of the $M$ subsets.

As a drawback of the proposed approach, individual models must be build because the level of spinal cord injury, fitness, body configuration, experience in FES usage, and approach to the standing-up, all contribute to the variability of COM trajectory between paraplegic subjects: every new subject included into the group changed the training data set. Thus, it is not reasonable to expect that a single model would successfully generalize over a bigger group of subjects. On the other side, the implementation of individual models requires measurement and a training procedure for each particular subject prior to model-making. This requirement can represent a serious obstacle in clinics where no kinematic measurement system is available. An embedded FES control system on which both the training and control algorithms would run would be a practical solution of this problem. Instead of a complex motion analysis system, a set of goniometers could be used to estimate the COM motion kinematics. With such a wearable system, incorporating force sensors and goniometers directly wired to the controller, adaptation could take place during standing-up exercises for each subject. The controller could include the online model training feature, and in this way, train the model automatically. Practicing for longer in the system adaptation phase would allow the changes in body dynamics due to muscle fatiguing to be incorporated in the model too.

In conclusion, the advantage of the proposed feedback system is clear when an estimate of the body COM trajectory during standing-up is required. This can be accomplished, at no cost to performance, from body support force signals, whose acquisition is not cumbersome for practical everyday usage.

\section{ACKNOWLEDGMENT}

The authors would like to thank Dr. N. Donaldson for his help during the final revision of the manuscript.

\section{REFERENCES}

[1] H. Gellman, I. Sie, and R. L. Waters, "Late complications of the weight bearing upper extremity in the paraplegic patient," Clin. Ortho. Relat. Res., vol. 233, pp. 132-135, 1998.

[2] A. Kralj and T. Bajd, Functional Electrical Stimulation: Standing and Walking After Spinal Cord Injury. Boca Raton, FL: CRC, 1989.

[3] T. Bajd, A. Kralj, and R. Turk, "Standing-up of a healthy subject and a paraplegic patient," J. Biomech., vol. 15, pp. 1-10, 1982.

[4] R. Kamnik, T. Bajd, and A. Kralj, "Functional electrical stimulation and arm supported sit-to-stand transfer after paraplegia: a study of kinetic parameters," Artif. Org., vol. 23, pp. 413-417, 1999.

[5] A. J. Mulder, P. H. Veltink, and H. B. K. Boom, "On/off control in FESinduced standing up: a model study and experiments," Med. Biol. Eng. Comput., vol. 30, pp. 205-212, 1992.

[6] M. J. Dolan, B. J. Andrews, and P. H. Veltink, "Switching curve controller for FES-assisted standing up and sitting down," IEEE Trans. Rehab. Eng., vol. 6, no. 2, pp. 167-171, Jun. 1998.

[7] J. Kuželički, R. Kamnik, T. Bajd, P. Obreza, and H. Benko, "Paraplegics standing-up using multichannel FES and arm support," J. Med. Eng. Technol., vol. 26, pp. 106-110, 2002.

[8] S. Heinze, P. H. Veltink, P. L. Jensem, R. E. Mayagoitia, H. J. Hermens, H. F. J. M. Koopman, and P. A. Huijing, "Development of control strategies for FES-supported closed-loop standing-up," in Neuroprosthetics From Basic Research to Clinical Applications, A. Pedotti, M. Ferrarin, J. Quintern, and R. Riener, Eds. Berlin, Germany: Springer-Verlag, 1996, pp. 209-216.

[9] D. J. Ewins, P. N. Taylor, S. E. Crook, R. T. Lipczynski, and I. D. Swain, "Practical low cost stand/sit system for mid-thoracic paraplegics," $J$ Biomed. Eng., vol. 10, pp. 184-188, 1988.

[10] R. Davoodi and B. J. Andrews, "Computer simulation of FES standing up in paraplegia: A self-adaptive fuzzy controller with reinforcement learning," IEEE Trans. Rehab. Eng., vol. 6, pp. 151-161, 1998.

[11] N. de N. Donaldson and C. H. Yu, "FES standing: Control by handle reactions of leg muscle stimulation (CHRELMS)," IEEE Trans. Rehab. Eng., vol. 4, no. 4, pp. 280-284, Dec. 1996.

[12] R. Riener and T. Fuhr, "Patient-driven control of FES-supported standing up: A simulation study," IEEE Trans. Rehab. Eng., vol. 6, no. 2, pp. 113-124, Jun. 1998.

[13] N. de N. Donaldson and C. H. Yu, "Experiments with CHRELMS patient-driven stimulator controllers for the restoration of function to paralyzed legs," Proc. Inst. Mech. Eng., H J. Eng. Med., vol. 214, pp. 1-20, 2000.

[14] R. Riener, M. Ferrarin, E. E. Pavan, and C. A. Frigo, "Patient-driven control of FES-supported standing up and sitting down: Experimental results," IEEE Trans. Rehab. Eng., vol. 8, no. 4, pp. 523-529, Dec. 2000. 
[15] M. K. Haugland, J. A. Hoffer, and T. Sinkjaer, "Skin contact force information in sensory nerve signals recorded by implanted cuff electrodes," IEEE Trans. Rehab. Eng., vol. 2, no. 1, pp. 18-22, Mar. 1994.

[16] J. P. Scholz and G. Schöner, "The uncontrolled manifold concept: Identifying control variables for a functional task," Exp. Brain Res., vol. 126, pp. 289-306, 1999.

[17] H. M. Toussaint, D. A. C. M. Commissaris, M. J. M. Hoozemans, M. J. Ober, and P. J. Beek, "Anticipatory postural adjustments before load pickup in a bi-manual whole body lifting task," Med. Sci. Sports Exerc., vol. 29, pp. 1208-1215, 1997.

[18] Y. Jian, D. A. Winter, M. G. Ishac, and L. Gilchrist, "Trajectory of the body COG and COP during initiation and termination of gait," Gait Post., vol. 1, pp. 9-22, 1993.

[19] Y. C. Pai, B. J. Naughton, R. W. Chang, and M. W. Rogers, "Control of body centre of mass momentum during sit-to-stand among young and elderly adults," Gait Post., vol. 2, pp. 109-116, 1994.

[20] A. B. Schultz, N. B. Alexander, and J. A. Ashton-Miller, "Biomechanical analyzes of rising from a chair," J. Biomech., vol. 25, pp. 1383-1391, 1992.

[21] P. J. Millington, M. Myklebust, and G. M. Shambes, "Biomechanical analysis of the sit-to-stand motion in elderly persons," Arch. Phys. Med. Rehab., vol. 73, pp. 609-617, 1992.

[22] A. Kralj, R. J. Jaeger, and M. Munih, "Analysis of standing up and sitting down in humans: Definitions and normative data presentation," $J$. Biomech., vol. 23, pp. 1123-1138, 1990.

[23] M. P. Murray, A. Seireg, and R. C. Scholz, "Center of gravity, center of pressure, and supportive forces during human activities," J. Appl. Physiol., vol. 23, pp. 831-838, 1967.

[24] D. A. Winter, "Estimations of the horizontal displacement of the total body centre of mass: Considerations during standing activities," Gait Post., vol. 1, pp. 141-144, 1993.

[25] P. De Leva, "Adjustments to Zatsiorksy-Seluyanov's segment inertia parameters," J. Biomech., vol. 29, pp. 1223-1230, 1996.

[26] B. J. Benda, P. O. Riley, and D. E. Krebs, "Biomechanical relationship between center of gravity and center of pressure during standing," IEEE Trans. Rehab. Eng., vol. 2, no. 1, pp. 3-9, Mar. 1994.

[27] D. Lenzi, A. Cappello, and L. Chiari, "Influence of body segment parameters and modeling assumptions on the estimate of center of mass trajectory," J. Biomech., vol. 36, pp. 1335-1341, 2003.

[28] S. Haykin, Neural Networks: A Comprehensive Foundation, 2nd ed. Englewood Cliffs, NJ: Prentice-Hall, 1999.

[29] C. K. I. Williams, "Prediction with Gaussian processes: from linear regression to linear prediction and beyond," in Learning and Inference in Graphical Models, M. I. Jordan, Ed. Dordrecht, The Netherlands: Kluwer, 1998, pp. 599-621.

[30] D. J. C. Mackay, "Introduction to Gaussian processes," in Neural Networks and Machine Learning. ser. NATO ASI, C. M. Bishop, Ed. Dordrecht, The Netherlands: Klewer, 1998, pp. 133-166.

[31] J. Q. Shi, R. Murray-Smith, and D. M. Titterington, "Hierarchical Gaussian process mixtures for regression," Stat. Comput., vol. 15, pp. $31-41,2005$.

[32] C. E. Rasmussen, "Evaluation of Gaussian processes and other methods for non-linear regression," Ph.D. dissertation, Graduate Dept. Comput. Sci., Univ. Toronto, Toronto, ON, Canada, 1996.

[33] T. Hastie, R. Tibshirani, and J. Friedman, The Elements of Statistical Learning. Berlin, Germany: Springer-Verlag, 2001.

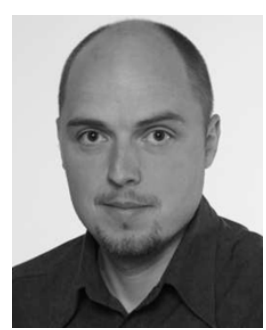

Roman Kamnik (M'98) received the B.Sc., M.Sc., and D.Sc degrees from the Faculty of Electrical Engineering, University of Ljubljana, Ljubljana, Slovenia, in 1992,1995 , and 1999 , respectively.

He was a Research Assistant with the Faculty of Electrical Engineering, University of Ljubljana, from 1992 to 1999 , and a Postdoctoral Research Fellow with the Department of Mechanical Engineering, University of Glasgow, Glasgow, U.K., in 2000. In 1998, he was also a Visiting Research Fellow with the Faculty of Medicine and Oral Health Sciences, Department of Biomedical Engineering, University of Alberta, Edmonton, $\mathrm{AB}$, Canada. He is currently an Assistant Professor of biomechanics with the Faculty of Electrical Engineering, University of Ljubljana. His research interests include the analysis and synthesis of multibody systems motion with the applications to biomedical engineering, robotics, and transport.

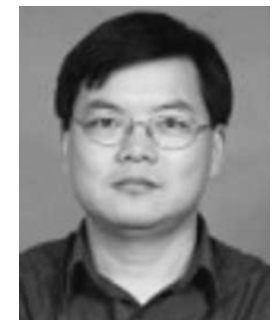

Jian Qing Shi received the B.Sc. degree in computing mathematics from Nanjing University, Nanjing, China, in 1984, the M.Sc degree in statistics from Southeast University, Nanjing, China, in 1990, and the Ph.D. degree in statistics from the Chinese University of Hong Kong, Hong Kong, in 1996.

He was a Postdoctoral Research Fellow with the Department of Statistics, University of Warwick, Coventry, U.K., from 1996 to 2000, and also with the Department of Computing Science, University of Glasgow, Glasgow, U.K., from 2000 to 2002 . He is currently a Lecturer of statistics with the School of Mathematics and Statistics, University of Newcastle, Newcastle, U.K. His research interests include nonparametric curve fitting, nonlinear system controls, meta-analysis, and covariance structural analysis.

Dr. Shi is a Fellow of the Royal Statistical Society.

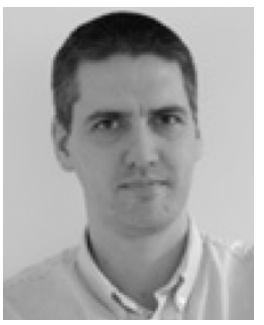

Roderick Murray-Smith received the B.Eng and $\mathrm{Ph} . \mathrm{D}$. degrees from the University of Strathclyde, Strathclyde, U.K. in 1990 and 1994, respectively.

$\mathrm{He}$ worked at Daimler-Benz Research Labs, Berlin, Germany, from 1990 to 1997 . He was a Visiting Researcher with the Department of Brain and Cognitive Sciences, Massachusetts Institute of Technology, Cambridge, in 1994, and a Research Fellow with the Department of Mathematical Modeling, Technical University of Denmark, Lynbgys, from 1997 to 1999. Since 1999, has been with the Department of Computing Science, Glasgow University, Glasgow, U.K. In 2001, he took up a joint position with the Hamilton Institute, National University of Ireland, Maynooth.

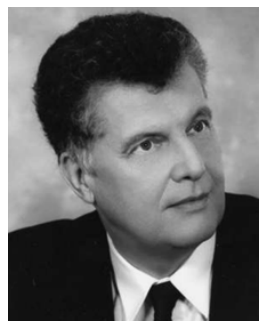

Tadej Bajd (A'87-SM'91-F'00) received Dipl.Ing., M.Sc., and D.Sc. degrees from Faculty of Electrical Engineering, University of Ljubljana, Slovenia, in 1972, 1976, and 1979, respectively.

He was a Research Assistant with the J. Stefan Institute, Ljubljana (1972-1977); and a Visiting Research Fellow with the University of Southern California, Los Angeles (1981) and Strathclyde University, Glasgow, U.K. (1984). He is currently a Professor of robotics with the Faculty of Electrical Engineering, University of Ljubljana, where he has also served as Vice-Dean (1985-1989) and Dean (1999-2003). He is author and coauthor of over 90 journal papers and two monographs on functional electrical stimulation and robot mechanisms. He is member of editorial board of several international and national journals.

Professor Bajd is President of Slovenian Society for Medical and Biological Engineering and is an Associate Member of Slovenian Academy of Sciences and Arts. He was awarded for his scientific achievements in the field of functional electrical stimulation for paralyzed subjects several times. 